\title{
Implications of high density operation on SOL transport: a multimachine investigation
}

\author{
D. Carralero ${ }^{\mathrm{a}^{*}}$, H.W. Müller ${ }^{\mathrm{a}}$, M. Groth ${ }^{\mathrm{b}}$, M. Komm ${ }^{\mathrm{c}}$, J. Adamek ${ }^{\mathrm{c}}$, G. Birkenmeier ${ }^{\mathrm{a}}$, \\ M. Brix ${ }^{\text {d }}$, F. Janky ${ }^{\text {c }}$, P. Hacek ${ }^{\text {c }}$, S. Marsen ${ }^{\text {d,e }}$, F. Reimold ${ }^{\mathrm{a}}$, C. Silva ${ }^{\mathrm{f}}$, U. Stroth ${ }^{\mathrm{a}}$, M. Wischmeier ${ }^{\mathrm{a}}$, \\ E. Wolfrum ${ }^{\mathrm{a}}$, ASDEX Upgrade Team ${ }^{\mathrm{a}}$, COMPASS Team ${ }^{\mathrm{c}}$ and JET-EFDA Contributors ${ }^{\mathrm{d}}$ \\ ${ }^{a}$ Max Planck Institute for Plasma Physics, Boltzmannstr. 2, 85748 Garching, Germany \\ ${ }^{b}$ Aalto University, Espoo, Finland. \\ ${ }^{c}$ Institute of Plasma Physics AS CR, IPP.CR, Praha 8, Czech Republic. \\ ${ }^{d}$ JET-EFDA, Culham Science Center, UK. \\ ${ }^{e}$ Max Planck Institute for Plasma Physics, Greifswald, Germany. \\ ${ }^{f}$ Institute of Plasmas and Nuclear Fusion, IST, Lisbon, Portugal.
}

\begin{abstract}
The transition reported in L-mode plasmas leading to the formation of a density "shoulder" in the Scrape-off Layer (SOL) of tokamaks is investigated in the three ITER stepladder machines: COMPASS, ASDEX Upgrade (AUG) and JET. In AUG and JET, the broadening of the profiles can be observed at similar relative densities $\left(\mathrm{n}_{\mathrm{e}} / \mathrm{n}_{\mathrm{GW}} \sim 0.4-0.5\right)$ and coincides with a clear increase in the size of SOL filaments and the onset of the outer divertor detachment. In COMPASS, no transition is observed in the same density range: the divertor does not detach and no clear effect is observed neither in the profiles nor in the turbulence. This is explained as the result of an increase of intermittent transport triggered by the increased resistivity along filaments associated to divertor detachment. The transition conditions in AUG and JET are compared to foreseen ITER SOL parameters, and the implications are briefly discussed.
\end{abstract}

PACS: 52.55.-s, 52.55.Fa, 52.35.Ra, 52.55.Rk

PSI-20 keywords: ASDEX-Upgrade, Detachment, Edge plasma, Intermittent transport, JET

*Presenting/corresponding author address: Boltzmannstrasse, 2 D-85748 Garching, Germany.

*Presenting/corresponding author E-mail: daniel.carralero@ipp.mpg.de 


\section{Introduction}

Heat and particle fluxes on Plasma-Facing Components (PFC) are one of the main concerns for the next generation of magnetic confinement fusion devices [1], which will almost certainly feature power loads and erosion levels on the verge of the technical limits of available plasmafacing materials. In order to predict reliably the distribution of such fluxes between the main chamber and the divertor, a proper understanding of transport processes in the Scrape-off Layer (SOL) is required. One important open question is the broadening of L-mode SOL profiles observed in many tokamaks (sometimes referred to as "shoulder") when density is increased over a certain threshold [2,3]. A recent work carried out in ASDEX Upgrade (AUG) [4] showed how this broadening was associated to a regime transition on SOL filaments, and to the beginning of the outer divertor detachment. As a result of it, density and power e-folding lengths, $\lambda_{\mathrm{n}}$ and $\lambda_{\mathrm{q}}$, were increased by a factor 5 and 3, respectively, and the average convected particle flux associated to filaments was increased by an order of magnitude. These results are consistent with a change from conduction to advection dominated transport, which has been proposed in the literature as an explanation for the "shoulder" [5]. According to this model, the increased collisionality caused either by the increased densities along the field line or the localized cooling of the divertor region taking place during the detachment would lead to a change on the closure scheme of filaments thus increasing perpendicular over parallel transport ratio. This would lead to a feedback loop, reducing the temperatures downstream along the field line and further increasing the collisionality.

The onset of a similar mechanism in the SOL of ITER could be of great practical importance, since it could substantially increase the particle flux arriving to the first wall, as well as contribute to spread particle and power fluxes on the divertor target. Both effects would have relevant consequences on the sputtering yield of the PFCs of both regions, thus changing the predicted life 
times of several components. In this work, the high density transition has been analyzed in the other two tokamaks of the so called ITER-stepladder (differently sized machines which have the same basic geometry as ITER): COMPASS and JET. The purpose of this multimachine comparison is to study the scaling of this transition and allow an extrapolation to ITER. With this aim, the evolution of midplane density profiles, filament characteristics and divertor detachment are measured in the three tokamaks. Afterwards, the results are compared, and the likelihood of a density transition in ITER is discussed by comparing AUG and JET SOL parameters in the experiments with projected values for ITER.

\section{Experiments}

In the three machines, L-mode plasmas with increasing densities and constant magnetic parameters and heating powers have been observed. All three experiments feature a similar geometry in the edge at the LFS, where filaments are supposed to be generated. On the contrary, the geometry of the divertor is different: COMPASS has an open divertor while AUG and JET feature closed ones. The strike points of both divertor legs at AUG lay on the vertical targets, while the LFS strike point of JET lays on the horizontal plate. In order to carry out a standardized comparison between the different machines, the Greenwald fraction, $\mathrm{f}_{\mathrm{GW}}=\mathrm{n}_{\mathrm{c}} / \mathrm{n}_{\mathrm{GW}}$, will be used through this work as a measure of plasma density, where $n_{c}$ is the core line averaged interferometer density and $\mathrm{n}_{\mathrm{GW}} \sim \mathrm{I}_{\mathrm{p}} / \pi \mathrm{a}^{2}$ is the Greenwald density [20], $\mathrm{I}_{\mathrm{p}}$ is the plasma current and $\mathrm{a}$ is the minor radius.

\subsection{ASDEX Upgrade}

The experiment carried out in AUG has been detailed in a previous work [4]. In it, several Lmode discharges (\#89321-26 \& \#89887) were carried out with increasing flattop densities in the 
range of $\mathrm{f}_{\mathrm{GW}} \in[0.15,0.6]$. The parameters of all discharges were: toroidal field at the magnetic axis $\mathrm{B}_{\mathrm{T}}=2.5 \mathrm{~T}$, plasma current $\mathrm{I}_{\mathrm{p}}=800 \mathrm{kA}$, safety factor $\mathrm{q}_{95}=5.32$, average triangularity $\delta=$ 0.235 , heating power $\mathrm{P}_{\mathrm{H}} \sim 1 \mathrm{MW}(500 \mathrm{~kW} \mathrm{ECH}+500 \mathrm{~kW}$ ohmic $)$ and $\mathrm{n}_{\mathrm{GW}}=1.02 \cdot 10^{20} \mathrm{~m}^{-3}$. The main diagnostics [6,7] are a 14 pin probe head installed on a fast reciprocator (RCP) used to measure the characteristics of SOL turbulence on the midplane $(0.31 \mathrm{~m}$ above the equator), the Lithium Beam (LiB) and the flush mounted Langmuir probes in the divertor plates (DPR).

As mentioned before, the main result of the experiment was the existence of a density threshold above which the density SOL broadens, filaments become larger and denser and overall perpendicular transport is increased [4]. In Fig. 1, the density profile as measured by the LiB is displayed for the whole range of densities. The radial resolution of the diagnostic is around $5 \mathrm{~mm}$. As can be seen, a clear change happens between $\mathrm{f}_{\mathrm{GW}} \sim 0.45-0.5$ : a flat region appears at the far SOL, which comes closer to the separatrix as $\mathrm{f}_{\mathrm{GW}}$ is increased. As a result, the density over the whole region is strongly increased. Besides, in Fig. 2a, it can be seen how this $\mathrm{f}_{\mathrm{GW}}=0.5$ coincides with the value at which the Degree of Detachment $(\mathrm{DoD})$ goes over DoD $=1$, indicating the onset of the LFS divertor detachment. This is confirmed by LFS divertor $\mathrm{I}_{\text {sat }}$ profiles (displayed in Fig. 10 of [4]), which show the onset of detachment for the same densities. It must be noticed that the calculation of the DoD has been done using the extrapolation method proposed by Loarte et al. [8], in which an empirical 2-point model curve is fitted over different discharges, rather the one used in [4], where a reference attached condition was found for each discharge. This change is made in order to compare with JET, where the DoD was calculated following the first method. Nevertheless, the results obtained with both methods are very similar. 


\subsection{JET}

JET experiments were carried out in December 2012, as part of an investigation on the effect of the ITER-like wall on divertor detachment [9]. The discharges used in this work (the JPN8147291 series) were conducted at machine parameters typical for JET in normal current and field direction: $\mathrm{I}_{\mathrm{p}}=2.5 \mathrm{MA}, \mathrm{B}_{\mathrm{T}}=2.5 \mathrm{~T}, \delta=0.255$ and $\mathrm{q}_{95}=3.4$. In them, different constant density flattops are achieved by changing the $\mathrm{D}$ puffing covering a density range of $\mathrm{f}_{\mathrm{GW}} \in[0.2,0.6]$. These densities cover all regimes of the divertor. As before, $\mathrm{PH}$ was kept constant at 3MW, from which 1.6 MW were injected by neutral beams and the rest were ohmic. In these low triangularity discharges, the HFS divertor leg lays on the vertical plate, and the LFS on the central horizontal plate [9]. D was puffed from the top of the machine in low density discharges, while for the highdensity plasma the inner baseplate injectors were also needed. The impact of the puffing location on the results is not expected to be significant, as recycling typically dominates gas injection. In this sense, experiments carried out in $\mathrm{H}$-mode show small variation on pedestal density and temperatures for magnetic configurations in which the outer strike point is away from the divertor duct pump (as in this case) [21]. The main diagnostics used in this work are again a RCP with a multipin probe [10], a LiB system [11] and an array of divertor probes. However, a couple of differences must be taken into account: first, the multipin probe could only measure radial correlations between ion saturation current $\left(\mathrm{I}_{\mathrm{sat}}\right)$ channels. Therefore, the polodial velocity and size of filaments can't be measured as in AUG, where the 14 pin probe allowed for correlations in the two perpendicular directions [4,6]. Second, the RCP and LiB are not installed in the outboard midplane, but in the upper diagnostic port of JET, probing the plasma from above [11]. The area observed by both diagnostics corresponds to a vertical stripe at $\mathrm{R}=3.25 \mathrm{~m}$ and a poloidal angle of $78^{\circ}$ in the JPN814xx series [9]. In this case, the spatial resolution of the LiB is $12 \mathrm{~mm}$ in $\mathrm{Z}$ at the measurement location, which translates into $5 \mathrm{~mm}$ at the outer midplane. 
In Fig. 1, the projection of the LiB density profiles on the outer midboard plane (OMP) is represented for the whole range of $\mathrm{f}_{\mathrm{GW}}$. The evolution is remarkably similar to the one observed in AUG: as $\mathrm{f}_{\mathrm{GW}}$ is increased, the gradient decreases and the density rises in the whole SOL. In this case, the transition is smoother than in AUG, but a change in profile shape becomes apparent after $\mathrm{f}_{\mathrm{GW}} \sim 0.4$, lower than the one found in AUG. Interestingly, a similar shift can be observed in Fig. 2, where the detachment of the LFS divertor of JET takes place for $\mathrm{f}_{\mathrm{GW}}=0.42$.

\subsection{COMPASS}

Discharges in COMPASS were designed to provide a third experiment with lower minor radius: a series of L-mode ohmic plasmas in which the flattop density is increased on a shot-to-shot basis to cover a range of densities $\mathrm{f}_{\mathrm{GW}} \in[0.15,0.53]$. This range is limited by the highest operational central line averaged density of COMPASS, around $n_{c}=10^{20} \mathrm{~m}^{-3}$. The main plasma parameters are $\mathrm{I}_{\mathrm{p}}=170 \mathrm{kA}, \mathrm{B}_{\mathrm{T}}=1.15 \mathrm{~T}$, and $\mathrm{q}_{95}=5$. The ohmic heating was kept constant at $\mathrm{P}_{\mathrm{H}}=250 \mathrm{~kW}$. The LFS edge has been shaped as in AUG, which, given the D-shape of standard COMPASS plasmas yields a higher average triangularity $(\delta \sim 0.37)$. The main diagnostics are a midplane reciprocating probe and the divertor probes. The RCP has been used both for the measurement of turbulence properties and radial profiles of density. In the first case, the same 14-pin probe head used in AUG was employed [6]. In the second, a probe head including ball pen probes and Langmuir pins was used [12] to obtain a simultaneous measurement of $T_{e}$ and $I_{s a t}$, from which the $\mathrm{n}_{\mathrm{e}}$ profile is derived. A different series of equivalent discharges were carried out with each probe: the \#6230-82 series with the 14 pin probe, and discharges \#6893, \#6900 and \#6909 with the BPP. 
The divertor measurements come from an array of Langmuir pins (DPR) distributed along the bottom tiles of the open COMPASS divertor [13], and are configured to measure $\mathrm{I}_{\mathrm{sat}}$.

One significant difference between COMPASS operation with respect to the previous experiments is the fact that the onset of divertor detachment doesn't happen in the range of densities achieved in the experiments. One of the reasons for this could be the divertor geometry: it has been observed experimentally that detachment happens at higher densities in tokamaks with an open divertor, typically in the range of $\mathrm{f}_{\mathrm{GW}} \sim 0.6$ [13]. Other factors might be the differences between a carbon and tungsten divertor, the effect of the geometry on pumping, etc. In good agreement with the original ansatz, this lack of detachment coincides with the absence of the "shoulder" in the midplane: In Fig. 1, the density profiles measured by the RCP are displayed over the range of $\mathrm{f}_{\mathrm{GW}}$. As can be seen, no transition can be observed in the profiles: the small increase observed for $\mathrm{f}_{\mathrm{GW}} \sim 0.5$ is within the error bars and is not comparable to the clear effects observed in AUG and JET for the same densities. However, it could be a hint of the same process taking place for even higher densities, in which the divertor can also be expected to detach.

\section{Discussion}

Profile data displayed in Fig. 1 indicate that the onset of the density "shoulder" begins at $\mathrm{f}_{\mathrm{GW}}$ 0.4 and 0.5 for JET and AUG, respectively. This coincides with the LFS divertor detachment densities, as shown in Fig. 2a. Instead in COMPASS, where the divertor remains attached over the observed range of $\mathrm{f}_{\mathrm{GW}}$, no "shoulder" is observed. In order to determine the role of filamentary transport in this transition, the evolution of turbulence with $\mathrm{f}_{\mathrm{GW}}$ is analyzed. 
To remove the influence of the distance to the separatrix, $\Delta$, all AUG points are selected in the same range of $\Delta \epsilon[10,25] \mathrm{mm}$, corresponding to the nearest measurements available. In the case of JET this is not possible, since RCP measurements were carried out at different radial positions over the $\mathrm{f}_{\mathrm{GW}}$ range. Therefore, points corresponding to several $\Delta$ values are represented, using different symbols. In the case of COMPASS, all measurements are in the $\Delta \epsilon[15,30] \mathrm{mm}$ range. In Fig. $2 b$, the autocorrelation time, $\tau_{\mathrm{AC}}$, of the reference $\mathrm{I}_{\mathrm{sat}}$ pin of the $\mathrm{RCP}$ at each machine is represented as a function of $\mathrm{f}_{\mathrm{GW}}$. In good agreement with previous results [4], AUG data clearly departs after the detachment of the divertor, with $\tau_{\mathrm{AC}}$ increasing by a factor of $4-5$. In JET, data points closest to the separatrix $(\nabla, \Delta<15 \mathrm{~mm})$ display a very similar behaviour, but with the threshold shifted roughly by the same amount as the detachment: from a close pre-transition value of $\tau_{\mathrm{AC}} \sim 20 \mu \mathrm{s}$, it increases sharply to $\tau_{\mathrm{AC}} \sim 90 \mu \mathrm{s}$ around the $\mathrm{f}_{\mathrm{GW}} \sim 0.42$ mark. Data points corresponding to higher $\Delta(\vartheta, 15 \mathrm{~mm}<\Delta<30 \mathrm{~mm})$ seem to undergo the same evolution, but on a more gradual fashion. Highest density values $\left(\Delta, \mathrm{f}_{\mathrm{GW}}>0.5\right)$ could only be measured at even greater $\Delta$ values due to safety concerns related to the RCP operation. They follow the trend of previous data, and achieve higher $\tau_{\mathrm{AC}}$ values than those observed in AUG. Finally, COMPASS fluctuations show a very weak increasing trend with density, but remain in pre-transition values for the whole range of $\mathrm{f}_{\mathrm{GW}}$ and don't undergo any transition, in good agreement with the divertor detachment hypothesis.

In order to provide a more physically meaningful measure of the filaments, the correlation between different pins of the RCP probe heads has been used to calculate the velocity of the detected structures. Given the probe configuration used in JET, which only allowed for correlations between radially separated $I_{\text {sat }}$ pins, the "radial size" of the filaments $\delta_{\mathrm{r}}=\tau_{\mathrm{AC}} \cdot \mathrm{V}_{\mathrm{r}}$ has 
been used for the comparison between the three machines (instead of the perpendicular size used in the AUG study [4]). In Fig. $2 c, \delta_{\mathrm{r}}$ is represented as a function of $\mathrm{f}_{\mathrm{GW}}$, using the same symbols as before. As can be seen, the same general trend can be observed in AUG and JET, with their $\delta_{\mathrm{r}}$ values departing roughly after the detachment density. Again, JET data show a more gradual change, although the tendencies of both machines become closer than in Fig $2 \mathrm{~b}$, even despite the different radial positions. The similitude between values in both machines is remarkable, especially taking into account that JET filaments are not measured in the midplane, as in AUG. Instead, COMPASS data show no clear trend, and display significantly higher $\delta_{\mathrm{r}}$ values than the other machines, especially for low $\mathrm{f}_{\mathrm{GW}}$. This indicates that the radial velocities of COMPASS filaments follow a different scaling with $\mathrm{f}_{\mathrm{GW}}$ than the ones in the larger tokamaks. This could be caused by the different structure of the divertor of COMPASS, with an X-point only one $\mathrm{cm}$ above the tiles (especially for densities under $\mathrm{f}_{\mathrm{GW}}=0.4$, for which no clear two leg structure can be seen in the DPR), but also by the lower connection length or higher presence of neutrals in the SOL. Regarding the comparison of filament sizes between the three machines, it is worth noting that several studies $[14,15]$ have shown that there is no strong influence of $\mathrm{B}_{\mathrm{T}}$ or in filament size, and therefore the different magnetic fields should not be relevant for this discussion.

The first conclusion of this study is that the same transition reported in many other tokamaks [24] is observed in JET. Besides, this multimachine comparison seems to reinforce the link between the start of LFS divertor detachment and midplane transitions in the particle transport and filamentary regimes: In AUG and JET, changes take place after the detachment, while no clear changes are reported in COMPASS where the divertor remains unaffected. The transition observed in AUG filaments displays a clear threshold, while the one in JET is more gradual. One reason for this could be that filaments in JET are measured in a region where the ideal filament 
dynamic model [5] (with curvature parallel to the pressure gradient) is only partially applicable. On the other hand, this gradual evolution is consistent with the smoother transition of density profiles observed in JET. Besides, the fact that a similar triggering process is observed in JET and AUG despite their different divertor geometry, adds to the robustness of the link between midplane transition and divertor conditions. It must be pointed out that, when calculated over the integral of the fluxes on the divertor (as in this work), DoD $>1$ doesn't mean a total detachment, but only the beginning of the transition from high recycling to detachment: in order to trigger the transition, the $T_{e}$ drop caused by the partial detachment seems to suffice. Still, the question remains of what causes the change in the filamentary transport. According to the model in [5], the parameter regulating the regime transition is effective collisionality, $\mathrm{L}=\mathrm{v}_{\mathrm{ei}} \mathrm{L} / \mathrm{c}_{\mathrm{s}} \Omega_{\mathrm{i}} / \Omega_{\mathrm{e}}$, where $\mathrm{v}_{\mathrm{ei}}$ is the ion-electron collision rate, $\mathrm{c}_{\mathrm{s}}$ is the sonic speed, $\Omega_{\alpha}$ is the gyrofrequency of species $\alpha$ and $\mathrm{L}$ is the parallel characteristic length. According to the model, for $\Lambda<1$, the midplane is connected to the wall, but for $\Lambda>1$ filaments undergo a regime transition which strongly increases their radial advection. If $\mathrm{L}=\mathrm{L}_{\mathrm{c}}$ is assumed (where $\mathrm{L}_{\mathrm{c}}$ is the connection length from the midplane to the closest target) and midplane values of $T_{e}$ and $n_{e}$ are used (due to the lack of SOL measurements in JET, $T_{i}=T_{e}$ is assumed), a rough estimation of the effective collisionality along the whole field line, $\Lambda_{\text {mid }}$, is obtained. In AUG, $\Lambda_{\text {mid }}=1$ is achieved at the same line density as the beginning of divertor detachment [4]. Therefore, it is not clear whether the filaments are disconnected due to the increased collisionality along the field line (caused by the increased density at the midplane), due to the localized increase of the collisionality in front of the divertor due to the detachment, or due to both at the same time. This question is of paramount importance for ITER: in the first case, no enhanced transport is to be expected according to this model, since the SOL will be at much higher temperatures than in present day devices. However, if the transition is triggered by 
divertor collisionality, the same effect could be expected in ITER, where the divertor will be detached at least in the region closer to the separatrix.

In order to compare the contributions of each region, the effective collisionality of the divertor, $\Lambda_{\text {div }}$, is estimated taking divertor $T_{e}$ and $n_{e}$, and assuming that the relevant parallel length scales as $\mathrm{L} \sim \mathrm{R}$. This scaling yields reasonable results in AUG and JET as the cold region in front of the detached divertor extends to the X-point [4], [22]. Besides, since $\mathrm{L}_{c}$ scales as $\mathrm{R}$, this scaling should also be qualitatively valid to carry out projections for ITER. In Table 1, the values of the different parameters and $\Lambda$ coefficients are displayed for different $\mathrm{f}_{\mathrm{GW}}$ values of AUG and JET, covering all three regimes of the divertor (low recycling, high recycling and detached). Also, representative values for the semidetached baseline scenario of ITER are included for comparison. In all cases, values correspond to the field line with $\Delta=15 \mathrm{~mm}$ in the midplane. This position corresponds to the end of the near SOL [18] and is the zone where the flat region begins after the transition in AUG. In Fig. $3, \Lambda_{\text {mid }}$ and $\Lambda_{\text {div }}$ are represented as a function of $f_{\mathrm{GW}}$ : as previously observed, both coefficients cross the $\Lambda=1$ threshold at the same density in AUG (coinciding with the DoD $=1$ of the LFS divertor). However, in the case of JET, only $\Lambda_{\text {div }}$ crosses the line at the approximate $\mathrm{f}_{\mathrm{GW}}$ of the transition, while $\Lambda_{\text {mid }}$ remains under the threshold until much higher $\mathrm{f}_{\mathrm{GW}}$ values. This indicates that the disconnecting effect of the cool divertor is enough to trigger the transition, independently of the midplane conditions. In both cases, $\Lambda_{\text {div }}$ is irrelevant for low $\mathrm{f}_{\mathrm{GW}}$ values, becomes $\Lambda=1$ roughly at the transition and surpasses the value of $\Lambda_{\text {mid }}$ afterwards, indicating that the contribution of the collisionality at the small divertor region after the detachment is greater than the accumulated effect along the field line (marginally in the case of AUG, by an order of magnitude in the case of JET). 
Values estimated with ITER projections $\left(\Lambda_{\text {mid }}=0.17\right.$ and $\left.\Lambda_{\text {div }}=258\right)$ suggest that this situation will be escalated, and the relevance of divertor collisions in the detached region will be four orders of magnitude greater than that of the midplane. Despite the crude estimation, these results make apparent that SOL filaments in ITER will not be connected to the wall, at least in the region where the divertor is detached. A complete description of the implications of this is out of the scope of this work (AUG, JET and COMPASS data correspond to L-mode operation and the standard ITER scenario will be H-mode). However, since no large type-I ELMs will be allowed in ITER, and given the high values of $\Lambda_{\text {div }}$, it can be expected that a similar mechanism will exist for inter-ELM filaments and even small type-III ELMs not carrying enough energy to force a local re-attachment.

\section{Conclusions}

By comparing the evolution of the SOL in the three tokamaks of the ITER stepladder, a clear increase in the density width has been found in JET and AUG, coinciding with the onset of detachment of the LFS divertor. Also, an increase in transport is observed in both machines associated with a change in filaments, which become larger and faster. Instead, no major changes were found in COMPASS, where the divertor remains attached over the same $f_{\mathrm{GW}}$ range. The main implication from this comparison is that it is the relevant change in collisionality - which, according to our model, determines the filamentary perpendicular transport in the midplane takes place in the divertor and does not depend on the midplane conditions. This could have profound implications in ITER: so far, the possibility of major convection towards the first wall has been disregarded given the high temperatures expected in the SOL. However, if the determinant parameter is the collisionality in the divertor (as the results from AUG, JET and 
COMPASS suggest), the partial detachment of ITER will be enough to cause the onset of a convective region at the end of the near SOL. Therefore, this effect should be included in ITER SOL simulations and taken into account when calculating particle and power fluxes onto the main wall and divertor, as it could have profound implications in the sputtering rates and life times of several key PFCs.

\section{Acknowledgements}

This work was supported by EURATOM and carried out within the framework of the European Fusion Development Agreement. The views and opinions expressed herein do not necessarily reflect those of the European Commission.

\section{References}

[1] A. Loarte, M. Sugihara, M. Shimada et al., 22nd IAEA Fusion Energy Conference (2008)

[2] B. LaBombard, R. L. Boivin, M. Greenwald et al., Phys. Plasmas, 8, 2107 (2001)

[3] D.L. Rudakov, J.A. Boedo, R.A. Moyer et al., Nucl. Fusion, 45, 1589-1599 (2005)

[4] D. Carralero, G. Birkenmeier, H. W. Müller et al, Nucl. Fusion, in press (2014)

[5] J. R. Myra, D. A. Russell, and D. A. D’Ippolito, Phys. Plasmas, 13, 112502 (2006)

[6] B. Nold, G. D. Conway, T. Happel, et al., Plasma Phys. Control. Fusion, 52, 065005 (2010).

[7] M. Willensdorfer, G Birkenmeier, R Fischer et al., Plasma Phys. Control. Fusion, 56, 025008 (2014).

[8] A. Loarte, R. D. Monk, J. R. Martín-Solís et al., Nucl. Fusion, 38, 331 (1998).

[9] M. Groth, S. Brezinsek, P. Belo et al., Nucl. Fusion, 53, 093016 (2013). 
[10] C. Silva, G. Arnoux, S. Devaux et al. J. Nucl. Mater., 438, S189-193 (2013)

[11] M. Brix, D. Dodt, D. Dunai, et al., Rev. Sci. Instrum., 83, 10D533 (2012)

[12] J. Adamek, J. Horacek, J. Seidl, Contrib. Plasma Phys. 54, No.3, 279-284 (2014)

[13] V. Weinzettl, R. Panek, M. Hron et al., Fusion Eng. And Design, 86 1223-1231 (2011)

[13] N. Asakura, N. Hosogane, K. Itami et al. Journal of Nucl. Mat., 1999, 266-269, 182-188

[14] G. Birkenmeier, F. M. Laggner, M. Willensdorfer, et al. accepted at Plasma Phys.

Control. Fusion (2014)

[15] S. J. Zweben, B. D. Scott, J. L. Terry, et al. Phys. Plasmas 16, 082505 (2009)

[16] A.S. Kukushkin, H.D.Pacher, V.Kotov, et al., Fusion Engineering and Design 86 2865-2873 (2011)

[17] E.E. Mukhin, R.A. Pitts, P. Andrew, Nucl. Fusion, 54, 043007, (2014)

[18] A.S. Kukushkin , H.D. Pacher, SOL/Div ITPA, Amsterdam, (2009)

[19] D. Coster, private communication.

[20] M. Greenwald, J. L. Terry, S. M. Wolfe et al., Nucl. Fusion, 28, 2199 (1988)

[21] E. R Solano, P. Tamain, E. Delabie et al., 41st EPS Conference, Berlin (2014)

[22] A.G. Meigs, S. Brezinsek, M. Clever et al. Journal of Nucl. Mat., 2013, 438, S607-S611

\section{Figure Captions}

Figure 1: Density in the SOL vs. radial distance to the separatrix for the various levels of $\mathrm{f}_{\mathrm{GW}}$. Profiles are normalized to their value at the separatrix, indicated as a dashed black line. AUG and JET profiles are measured by the LiB, COMPASS profiles are measured by the BPP.

Figure 2: a) The degree of detachment (DOD) of AUG (red) and JET (blue) LFS divertors vs. $f_{\mathrm{GW}}$. b) Autocorrelation time of the midplane fluctuations vs. $f_{\mathrm{GW}}$ c) Radial size of filamentary structures vs. $\mathrm{f}_{\mathrm{GW}}$. Blue symbols in $\mathrm{b}$ ) and c) correspond to different vertical distances to JET separatrix: $(\nabla) \Delta<15 \mathrm{~mm},(\diamond) 15 \mathrm{~mm}<\Delta<30 \mathrm{~mm},(\Delta) \Delta>30$. The beginning of JET and AUG divertor detachment is indicated as a solid vertical line of the same color in all three figures. 
Figure 3: Effective collisionality parameter, $\Lambda$, for different $\mathrm{f}_{\mathrm{GW}}$ values at AUG (red) and JET (blue). Circles/squares indicate $\Lambda$ values at the separatrix/divertor. The $\mathrm{f}_{\mathrm{GW}}$ threshold for detachment is indicated as in Fig. 2.

Table 1: SOL parameters for AUG, JET and ITER.

Tables

\begin{tabular}{|c|c|c|c|c|c|c|c|c|c|}
\hline Machine & $\mathrm{R}(\mathrm{m})$ & $\mathrm{f}_{\mathrm{GW}}$ & $\mathrm{T}_{\mathrm{e}, \text { mid }}(\mathrm{eV})$ & $\mathrm{n}_{\mathrm{e}, \text { mid }}\left(\mathrm{m}^{-3}\right)$ & $\mathrm{T}_{\text {e,div }}(\mathrm{eV})$ & $\mathrm{n}_{\mathrm{e}, \text { div }}\left(\mathrm{m}^{-3}\right)$ & $\mathrm{L}_{\mathrm{c}}(\mathrm{m})$ & $\Lambda_{\text {mid }}$ & $\Lambda_{\text {div }}$ \\
\hline AUG & 1.65 & 0.15 & 15 & $1.70 \mathrm{E}+18$ & 20 & $6.00 \mathrm{E}+17$ & 20 & 0.25 & 0.00 \\
\hline & 1.65 & 0.45 & 15 & $4.20 \mathrm{E}+18$ & 7 & $1.00 \mathrm{E}+19$ & 20 & 0.62 & 0.54 \\
\hline & 1.65 & 0.55 & 15 & $1.20 \mathrm{E}+19$ & 5 & $1.50 \mathrm{E}+19$ & 20 & 1.78 & 2.03 \\
\hline JET & 2.96 & 0.25 & 25 & $3.00 \mathrm{E}+18$ & 10 & $1.00 \mathrm{E}+18$ & 32 & 0.25 & 0.05 \\
\hline & 2.96 & 0.35 & 25 & $7.50 \mathrm{E}+18$ & 5 & $4.00 \mathrm{E}+19$ & 32 & 0.64 & 7.92 \\
\hline ITER & 6.20 & 0.85 & $80^{[16]}$ & $1.3 \mathrm{E}+19^{[16]}$ & $2^{[17]}$ & $1 \mathrm{E}+20^{[17,18]}$ & $50^{[19]}$ & 0.17 & 258.52 \\
\hline
\end{tabular}

Table 1 
Figures
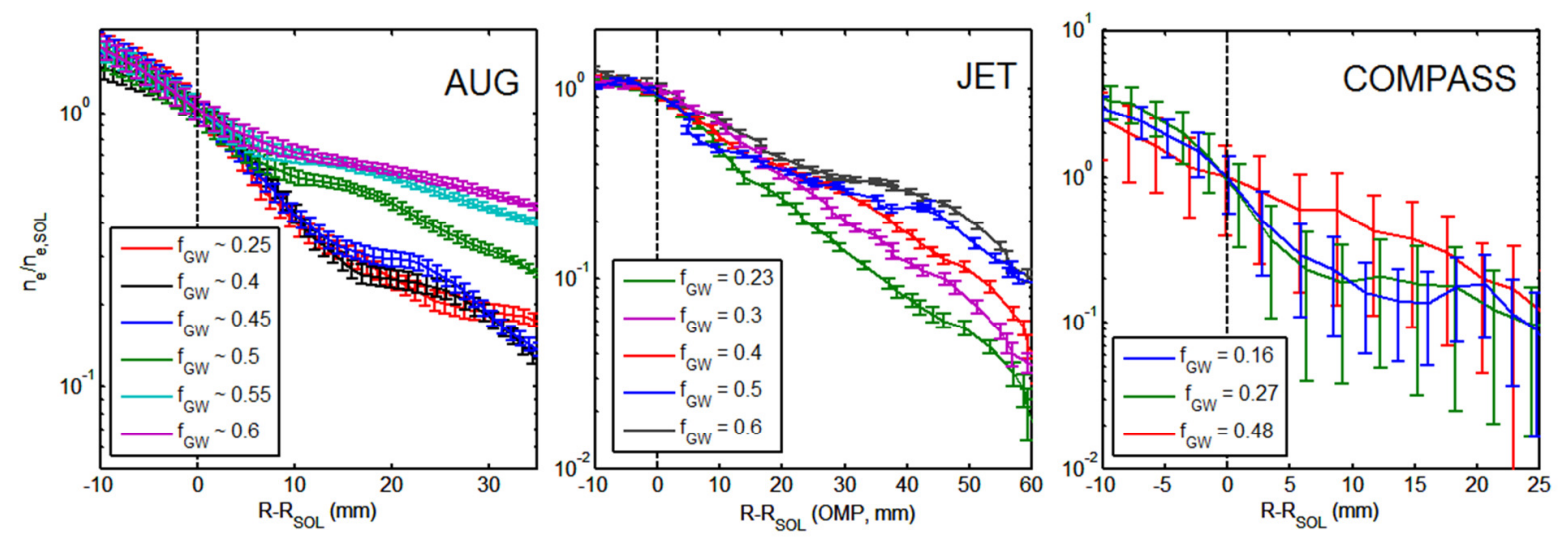

Figure 1 

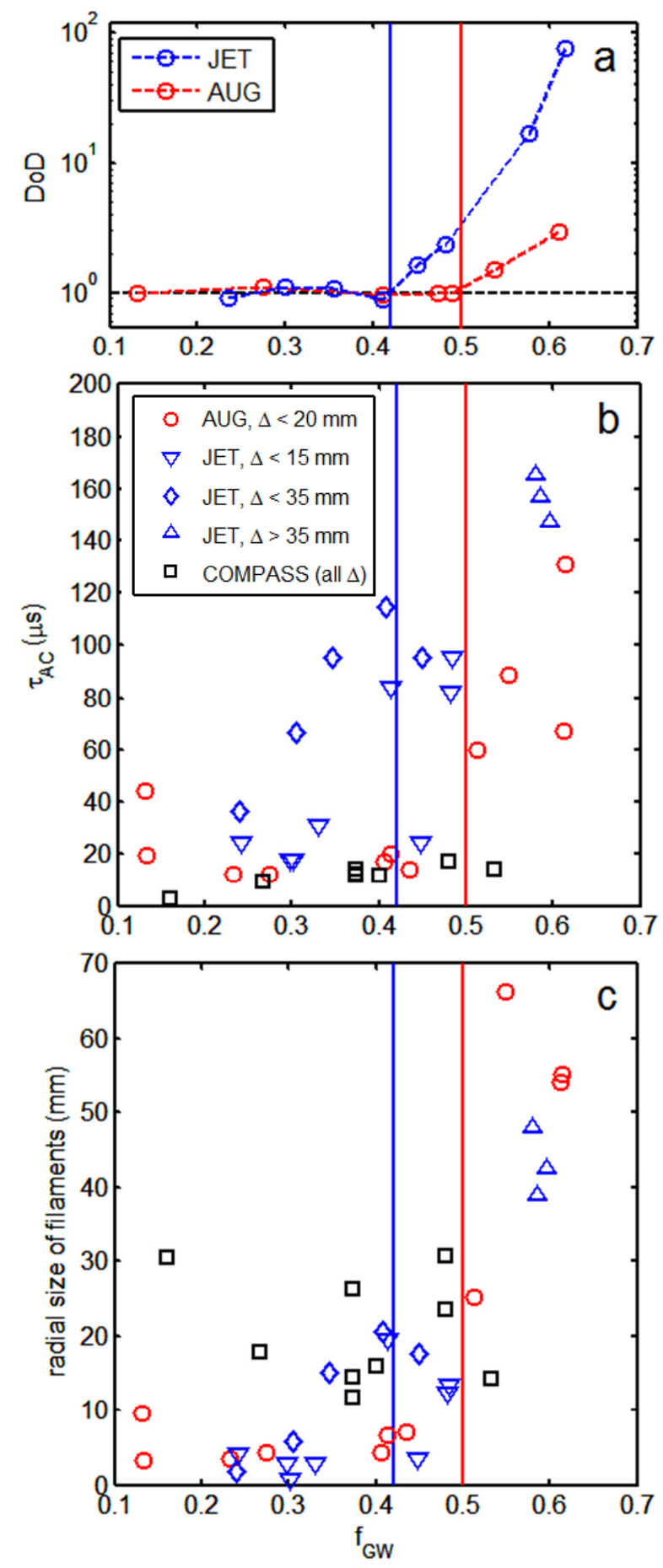

Figure 2 


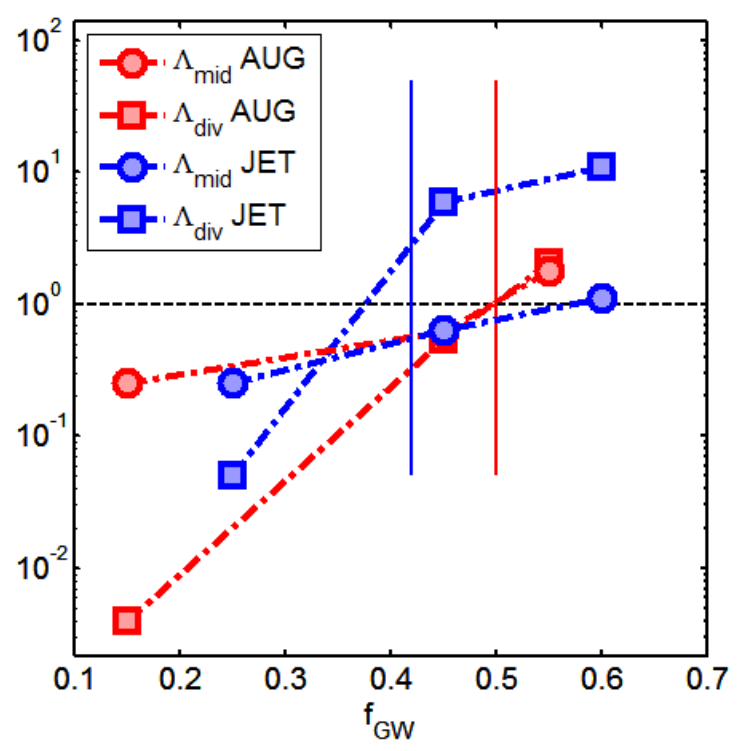

Figure 3 